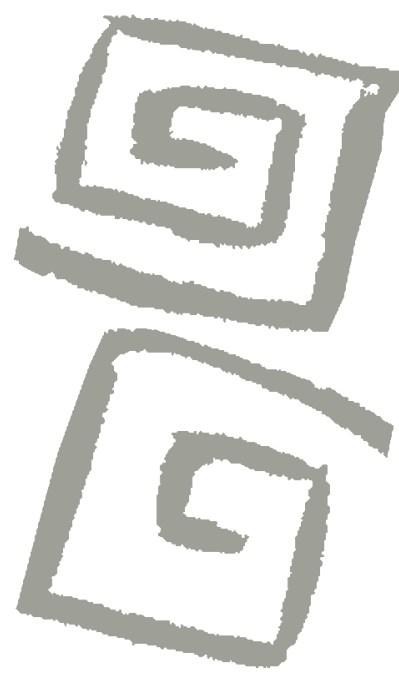

\title{
Estudio exploratorio de validez y consistencia interna del índice de capacidad para el trabajo entre trabajadores de la salud
}

\author{
Exploratory study of the validity and internal \\ consistency of the Work Ability Index among health \\ workers
}

Técia Maria Santos Carneiro e Cordeiro', Tânia Maria de Araújo², Kionna Oliveira Bernardes Santos ${ }^{3}$

'Enfermera. Magíster en Salud Colectiva. Investigadora, Núcleo de Epidemiologia, Universidade Estadual de Feira de Santana. Bahía, Brasil. $\triangle$ iD

${ }^{2}$ Psicóloga. Doctora en Salud Pública. Coordinadora, Núcleo de Epidemiologia. Docente, Universidade Estadual de Feira de Santana. Bahía, Brasil. $\triangle$ (iD

${ }^{3}$ Fisioterapeuta. Doctora en Salud Pública, Universidade Federal de la Bahia. Docente, Universidade Federal da Bahia. Bahía, Brasil. $\bowtie$ iD
RESUMEN El objetivo de este estudio fue evaluar la validez y la consistencia interna del índice de capacidad para el trabajo (ICT) entre trabajadores de la salud en Bahía, Brasil. Se trata de un estudio exploratorio de validez con base en un corte transversal de una muestra de 3.084 trabajadores de la salud. El análisis de componentes principales extrajo tres factores del instrumento con varianza total acumulada del 53,9\%. A través del ICT se identificaron grupos de trabajadores por edad y se correlacionaron de forma positiva con el control en el trabajo, el apoyo social y la autopercepción del estado de salud y, de forma negativa con los trastornos mentales comunes, la demanda psicológica, física y emocional y el desequilibrio esfuerzo-recompensa. El alfa de Cronbach subestimó los coeficientes de cada factor en comparación con el omega de McDonald. EI ICT presentó propiedades psicométricas satisfactorias, tanto para medir la capacidad física y mental para el trabajo en grupos de trabajadores por edad y correlacionar a los aspectos relacionados con la salud. PALABRAS CLAVES Salud Laboral; Evaluación de Capacidad de Trabajo; Personal de Salud; Reproducibilidad de los Resultados; Brasil.

\begin{abstract}
The objective of this study was to evaluate the validity and internal consistency of the Work Ability Index (WAI) among health workers in Bahia, Brazil. An exploratory validity study was conducted based on a cross-sectional sample of 3,084 health workers. The principal component analysis extracted three factors of the instrument with a total accumulated variance of $53.9 \%$. The WAI was able to identify groups of workers by age and show positive correlations with job control, social support and self-perceived health status as well as negative correlations with common mental disorders, psychological, physical and emotional demand and the effort-reward imbalance. The Cronbach's alpha underestimated the coefficients of each factor compared to McDonald's omega. The WAI showed satisfactory psychometric properties to measure physical and mental work ability in groups of workers according to age.
\end{abstract}

KEY WORDS Occupational Health; Work Capacity Evaluation; Health Personnel; Reproducibility of Results; Brazil. 


\section{INTRODUCCIÓN}

La capacidad para el trabajo es un constructo multidimensional que abarca precondiciones físicas y mentales del trabajador y aspectos organizacionales y ambientales del trabajo. Para su verificación se desarrolló el índice de capacidad para el trabajo (ICT), un instrumento que se refiere al bienestar del trabajador en el presente y en el futuro y a capacidad de ejecutar su trabajo en función de las exigencias, de su estado de salud y de sus capacidades físicas y mentales. Tiene, también, carácter predictivo y aborda la autopercepción del propio trabajador sobre su salud y capacidad para el trabajo ${ }^{(1)}$.

La base teórica del ICT fue el modelo del estrés-desgaste. Este modelo asocia el desgaste del trabajador como consecuencia de estresores físicos, mentales y psicosociales del trabajo, que son equilibrados por factores individuales del trabajador ${ }^{(2,3)}$. El ICT se utiliza en servicios de salud ocupacional como apoyo para acompañar, mantener y/o desarrollar la capacidad para el trabajo, además de apoyar a los trabajadores desde el ingreso en el trabajo ${ }^{(1)}$.

La complejidad del concepto de capacidad para el trabajo es un desafío para la investigación ${ }^{(1)}$ por su carácter subjetivo y por tratarse de un constructo multidimensional, verificado por instrumentos que también son subjetivos. Según una revisión sistemática(4), estudios de validez y reproductibilidad del ICT se desarrollaron en el mundo con trabajadores de enfermería hospitalaria ${ }^{(5,6)}$, metalúrgicos ${ }^{(7)}$, electricistas ${ }^{(8)}$, trabajadores de la construcción civil(9), enfermeras ${ }^{(10)}$, trabajadores de la salud hospitalaria ${ }^{(11)}$, trabajadores de la salud de atención primaria ${ }^{(12)}$, trabajadores de la industria naval ${ }^{(13)}$, trabajadores voluntarios $^{(14)}$ y trabajadores de diferentes grupos ocupacionales ${ }^{(15)}$.

La mayoría de los estudios que utilizaron las preguntas del índice ${ }^{(4)}$ presentaron la estructura factorial del ICT con tres factores, con una varianza acumulada del $57,6 \%$ entre electricistas ${ }^{(8)}$ al $66,0 \%$ en trabajadores de atención primaria de la salud ${ }^{(12)}$ y la con- sistencia interna fue verificada con el alfa de Cronbach, la cual varió del 0,58 en trabajadores de oficina(15) $^{(15)}$ a,80 en trabajadores de atención primaria de la salud ${ }^{12}$.

Aunque la validación del ICT se haya realizado en varios países y se haya confirmado su relevancia para el campo de la salud del trabajador, aún existen lagunas en el proceso de validación del ICT, las cuales se refieren a las técnicas usualmente empleadas para esa evaluación y los tipos de efecto focalizados. Así, este estudio buscó ampliar el alcance de las técnicas utilizadas en la evaluación. Además, se consideró, la realización de estudios de validez con grupos de trabajadores en diferentes ocupaciones, regiones, estados y municipios del país, los cuales presentan distintas características, condiciones de mensuración y variabilidad del cuestionario, lo que justifica y estimula la realización de estudios de validación entre diferentes poblaciones.

Cabe señalar que, en Brasil, no hay registro de estudio del ICT para evaluar la capacidad para el trabajo entre trabajadores de la salud de baja y media complejidad ${ }^{(4)}$. Algunos estudios $^{(5,6)}$ evaluaron solo trabajadores de enfermería en contexto hospitalario, aunque las características laborales de estos trabajadores son distintas, pues abarcan múltiples funciones que provocan sobrecarga de trabajo, además de bajos salarios que los obligan a tener múltiples vínculos laborales. Ante estas lagunas, el objetivo de este estudio fue evaluar la validez y la consistencia interna del índice de capacidad para el trabajo entre trabajadores de la salud en Bahía, Brasil.

\section{MÉTODOS}

\section{Diseño de estudio}

Se trata de estudio exploratorio de validación con base en una investigación de corte transversal de carácter multicéntrico "Condiciones de trabajo, condiciones de empleo y salud de los trabajadores de la salud de la atención básica en Bahía" desarrollado en cinco municipios de Bahía: Feira de Santana, 
Salvador (Distrito Sanitario Centro Histórico), Jequié, Santo Antônio de Jesus y Itabuna.

\section{Participantes}

La población-objetivo de este estudio fueron todos los trabajadores de la salud de las unidades de salud de baja y media complejidad de los municipios estudiados, considerando trabajadores de la salud a todos aquellos asentados en las unidades, como administrativos, porteros, médicos, dentistas, farmacéuticos, técnicos de salud bucal, enfermeros, asistentes sociales, servicios generales, agentes comunitarios de salud, entre otros trabajadores de este universo.

Se incluyeron aquellos trabajadores que tenían más de seis meses de servicio y que estaban en actividad en el momento de la investigación. Cada municipio adoptó técnicas de muestreo diferentes: Santo Antônio de Jesus, Salvador y Jequié adoptaron una técnica poblacional, y Feira de Santana e Itabuna, muestral. La muestra de estudio fue aleatoria, por medio de sorteo de los trabajadores a ser incluidos en cada región establecida y estratificada por área geográfica de alcance de los servicios.

Para estimar el tamaño muestral y la representatividad poblacional, se calculó una muestra que consideró una frecuencia del $31,3 \%$ de capacidad para el trabajo inadecuada, un error del $5 \%$ y reposición del $20 \%$, para garantizar la representatividad por las pérdidas, Ilegando a una muestra de 377 . Sin embargo, se entrevistaron 3.084 trabajadores de la salud, superando el tamaño muestral establecido.

De los 3.084 trabajadores de la salud que participaron del estudio, el $78,2 \%$ era mujer y el $21,8 \%$ hombre, el $63,5 \%$ tenía enseñanza media completa y la edad varió de 19 a 82 años con una media de $39,5 \pm 10,7$ años. En cuanto a las características ocupacionales, el $32,5 \%$ prestaba cuidados directos a los usuarios, el 30,3\% era agentes comunitarios de salud y el $37,2 \%$ realizaba otras tareas; el 65,3\% tenía hasta 10 años de trabajo en su función, el $77,7 \%$ indicó una jornada de trabajo semanal de hasta 40 horas y el $64,8 \%$ estaba contratado.

\section{Instrumentos}

Se utilizaron los siguientes cuestionarios: índice de capacidad para el trabajo (ICT) $)^{1,5}$, self reporting questionnaire (SRQ-20) ${ }^{16}$, effort-reward imbalance (ERI) ${ }^{17}$ y job content questionnaire (JCQ) ${ }^{18}$. EI ICT, objeto de investigación de este estudio, fue aplicado en la versión traducida y adaptada al portugués ${ }^{(1)}$, se trata de un instrumento de evaluación de la capacidad para el trabajo, compuesto por diez preguntas agrupadas en siete dimensiones: 1) capacidad para el trabajo comparada con la mejor de toda la vida (respuestas de 1-10, escala discreta); 2) capacidad para el trabajo con relación a las exigencias del trabajo, compuesta por dos preguntas: recursos físicos y recursos mentales (respuestas de 1-5; escala ordinal); 3) número de enfermedades actuales diagnosticadas por médico, con base en una lista de 51 enfermedades (respuestas de 0-51; escala discreta); 4) pérdida estimada para el trabajo por causa de enfermedades (respuestas de 1-6; escala politómica); 5) faltas al trabajo por enfermedades en el último año (respuestas de 1-5; escala ordinal); 6) pronóstico propio de la capacidad para el trabajo (respuestas 1,4 y 7 , escala politómica); 7) recursos mentales, ponderadas por medio de tres preguntas: valoración de las actividades diarias, activo y alerta, y esperanza para el futuro (respuestas de 0-4; escala de razón). La sumatoria de las puntuaciones varía entre 7 y 49 puntos (escala discreta). Con los puntuaciones se puede evaluar la capacidad para el trabajo en cuatro categorías: baja ( 7 a 27), moderada (28 a 36), buena (37 a 43) y óptima $(44 \text { a } 49)^{(1)}$.

El SRQ-20 ${ }^{(16)}$ es un instrumento que permite mensurar la sospecha de trastornos mentales comunes. Está compuesto por 20 preguntas referentes a las situaciones vividas en los últimos 30 días. Las preguntas son dicotómicas (sí-no) y la puntuación se obtiene por la suma de las respuestas, y puede variar de 0 a 20 en una escala cuantitativa discreta.

El ERI ${ }^{(17)}$ fue utilizado para verificar el estrés en el trabajo por medio de la escala de equilibrio esfuerzo-recompensa. Es un instrumento compuesto por 23 preguntas del tipo 
Likert que se agrupan en tres escalas: esfuerzo (seis preguntas), recompensa (11 preguntas) y exceso de compromiso (seis preguntas). La puntuación obtenido de las escalas por medio del cálculo [esfuerzo/(recompensa*factor de corrección)] varía entre 0 y 2 (escala discreta).

El JCQ ${ }^{(18,19)}$, instrumento compuesto por 49 preguntas con respuestas ordinales del tipo Likert, se utilizó para verificar aspectos psicosociales del trabajo: habilidad (seis preguntas) y decisión (tres preguntas), demanda psicológica (cinco preguntas), demanda física (cuatro preguntas), emocional (tres preguntas) y apoyo social de colegas (3 preguntas) y directivos (3 preguntas). Cada dimensión se calculó de acuerdo al manual del JCQ y las puntuaciones varían en una escala discreta.

La autopercepción del estado de salud fue mensurada por la siguiente pregunta: cómo considera su estado de salud en comparación con las personas de su edad. Las respuestas se presentan en una escala ordinal de 1 a 5 , de muy bueno a muy malo.

\section{Procedimentos}

La recolección de datos se realizó entre 2011 y 2012, en el propio lugar de trabajo, y estuvo a cargo de equipos de entrevistadores entrenados. Se utilizó un cuestionario previamente testeado en unidades de salud. Para los trabajadores de la salud de nivel superior, el cuestionario fue autoaplicado y para aqueIlos que tenían hasta enseñanza media se realizaron entrevistas.

\section{Análisis de datos}

La validez del constructo se llevó a cabo a través del análisis de componentes principales. Para eso se calculó el test de esfericidad de Bartlett $(p<0,05)$ y el test de Kaiser-MeyerOlkin para verificar la factibilidad, siendo los mejores resultados aquellos cercanos a $1^{(20)}$.

Se realizó, también, el análisis univariado de las preguntas del instrumento para verificar la sensibilidad psicométrica del ítem, a partir de la media y el desvío estándar, y de la asimetría y curtosis y sus respectivos errores. Los valores de la asimetría $>3$ y de la curtosis $>7$ fueron considerados como dificultades en la sensibilidad de los ítems.

Posteriormente, se realizó el test scree plot o test de Cattell para verificar la retención de los componentes (factores) a partir de los autovalores. Para el cálculo paralelo, se aplicó el procedimiento de simulación de Monte-Carlo, que compara los datos reales con los datos aleatorios, con el objetivo de observar los factores del instrumento a partir de los autovalores ${ }^{(20)}$.

Para la extracción de los factores se aplicó el método de componentes principales y el número de factores extraídos se consideró a través del criterio de Kaiser, que excluye aquellos con autovalores $<1$. Luego, se realizó la rotación oblicua Promax asumiendo la existencia de correlación entre los fatores ${ }^{(21)}$. Se consideraron las cargas factoriales aceptables $\geq 0,30^{(20,21)}$.

Se mensuraron también las comunalidades y los errores. Para las comunalidades se esperaban valores superiores a $0,5 \mathrm{y}$, para los errores, valores inferiores a $0,5^{(22)}$. Se verificó la multicolinealidad a través de las correlaciones entre cada factor y otros seleccionados de la matriz de correlaciones. Si las intercorrelaciones eran altas, por encima de 0,85, indicaba multicolinealidad entre los factores.

Para verificar la diferenciación del ICT se procedió a un análisis de cada factor extraído según los grupos de trabajadores por edad ( $<35$ años, 36 a 50 años y $>50$ años) y se compararan las diferencias entre los grupos por medio del test Kruskal-Wallis $(p<0,05)$. Se antecedeu-se con el test de KolmogorovSmirnov el cual presentó una distribución de los datos con anormalidad $(p<0,05)$.

La validez convergente o discriminante se midió con el test de correlación de Spearman $(p<0,05)$ estimado para verificar las correlaciones entre cada factor extraído del ICT y las escalas referentes a la salud física y mental, y los estresores ocupacionales verificados por el JCQ, ERI, SRQ-20 y autopercepción del estado de salud.

La consistencia interna fue calculada para los factores extraídos en el análisis 
Tabla 1. Media, asimetría y curtosis de cada pregunta del índice de capacidad para el trabajo. Bahía, 2011-2012.

$\begin{array}{lcccccccc}\text { Pregunta } & \mathrm{n} & \text { Media } & \mathrm{DE} & \text { Asimetría } & \mathrm{EE} & \text { Curtosis } & \text { EE } \\ \text { 1. Capacidad para el trabajo actual } & & & & & & & & \\ \text { 2. Exigencias físicas del trabajo } & 3.064 & 8,52 & 1,29 & -1,30 & 0,04 & 3,88 & 0,09 \\ \text { 3. Exigencias mentales del trabajo } & 3.064 & 3,72 & 0,88 & -0,59 & 0,04 & 0,56 & 0,09 \\ \text { 4. Número de enfermedades } & 3.064 & 4,02 & 0,79 & -0,80 & 0,04 & 1,38 & 0,09 \\ \text { 5. Faltas al trabajo debido a enfermedades } & 2.936 & 4,34 & 2,03 & -0,13 & 0,04 & -1,10 & 0,09 \\ \text { 6. Ausentismo } & 3.023 & 5,35 & 0,98 & -1,77 & 0,04 & 3,47 & 0,09 \\ \text { 7. Pronóstico de la capacidad para el trabajo } & 3.061 & 4,35 & 0,89 & -1,64 & 0,04 & 2,82 & 0,09 \\ \text { 8. Valoración de las actividades diarias } & 3.062 & 6,24 & 1,59 & -1,99 & 0,04 & 3,04 & 0,09 \\ \text { 9. Sentirse activo y alerta } & 3.061 & 3,14 & 0,97 & -0,84 & 0,04 & -0,17 & 0,09 \\ \text { 10. Esperanza para el futuro } & 3.063 & 3,38 & 0,86 & -1,30 & 0,04 & 1,11 & 0,09 \\ & 3.067 & 3,38 & 0,95 & -1,41 & 0,04 & 1,11 & 0,09\end{array}$

factorial por medio de los coeficientes alfa de Cronbach $(\alpha)$ y omega de McDonald $\left(\omega^{t}\right)^{(23)}$.

El análisis se realizó con los programas estadísticos Statistics Data Analisis STATA versión 10.0 y Statistical Package for Social Science (SPSS) versión 18.0. Este estudio fue aprobado por el Comité de Ética en Investigación institucional bajo el parecer $\mathrm{n}^{\circ} 846.062 / 2014 \mathrm{y}$ CAAE 33104714.8.0000.0053.

\section{RESULTADOS}

EI ICT presentó una puntuación media de 40,0 puntos ( $\mathrm{DP}=5,0$ puntos): $51,3 \%$ de los trabajadores presentaron una buena capacidad para el trabajo, 27,2\% óptima, 19,6\% moderada y $2,0 \%$ baja. La sensibilidad psicométrica fue observada en todas las preguntas del ICT, el que indica distribución poco divergente de una distribución normal (Tabla 1). Los tests de Kaiser-Meyer-Olkin (KMO) $(0,77)$ y el de esfericidad de Bartlett $\left(X^{2}=4592,99\right.$; $p<0,001)$ apoyaron la factibilidad de los datos. Con relación al scree plot y al análisis paralelo de los datos, los dos métodos indicaron la extracción de tres factores a partir del criterio de Kaiser (Figura 1).

En la validez de constructo por medio del análisis de los componentes principales, se observa que los tres factores explicaron el $53,9 \%$ de la varianza total acumulada. EI primer factor, que se refiere a los recursos mentales, explicó el 29,0\% de la varianza, y contenía las preguntas: 8) apreciación de las actividades diarias, 9) sentirse activo y alerta y 10) esperanza para el futuro. El segundo factor, que se refiere a la capacidad para el trabajo física y mental, explicó el 13,8\% de la varianza, y contenía las preguntas: 1) capacidad para el trabajo actual, 2) exigencias físicas del trabajo y 3) exigencias mentales del trabajo. El tercero factor, que se refiere a la presencia de enfermedades y restricción ante el estado de salud, explicó solo el 11,0\% de la varianza, y estuvo conformado por las preguntas: 4) número de enfermedades, 5) faltas al trabajo debido a enfermedades, 6) ausentismo y 7) pronóstico de capacidad para el trabajo (Tabla 2).

Las comunalidades y especificidades presentadas en las preguntas 1) capacidad para el trabajo actual, 6) ausentismo y 7) pronóstico de la capacidad para el trabajo indicaron un bajo poder explicativo por el factor correspondiente. La pregunta 7 presentó una carga factorial límite $(0,31)$ y el factor 3 presentó la menor varianza del componente $(11,0 \%)$. No se observaron problemas de multicolinealidad de las correlaciones entre los factores. 


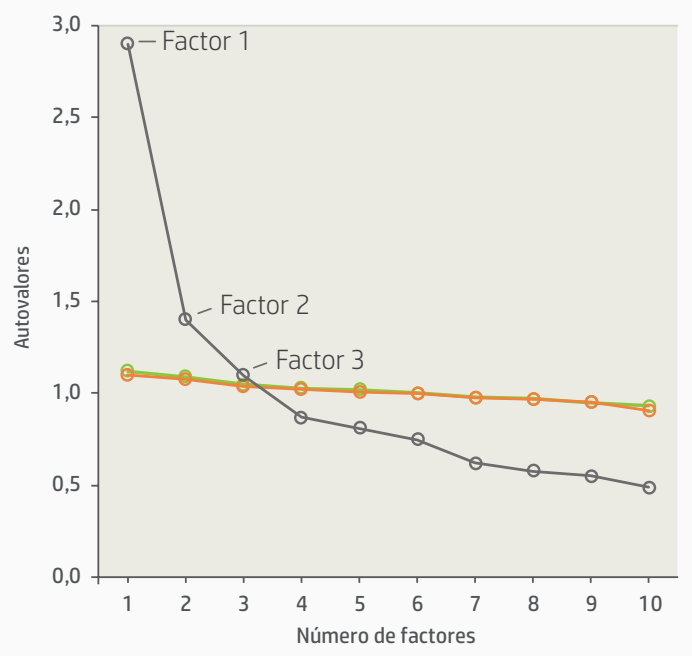

-Datos observados $\simeq$ Datos aleatorios $\simeq$ Datos remuestrados

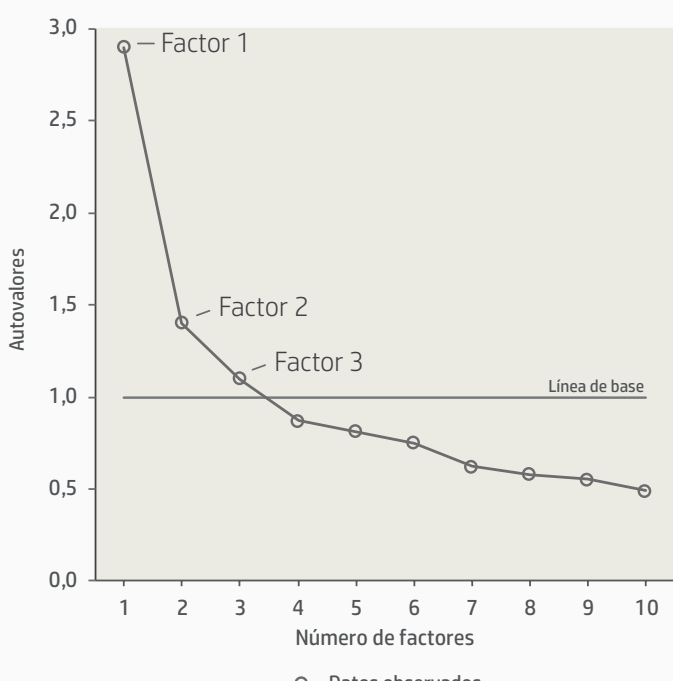

Datos observados

Figura 1. Valores de los componentes principales de los datos, según análisis paralelo y de autovalores de las preguntas del índice de capacidad para el trabajo. Bahía, Brasil, 2011-2012.

Fuente: Elaboración propia.

Línea de base: Representa el criterio de Kaiser que excluye los factores con autovalores $<1$.

Se observaron diferencias estadísticamente significativas $(p<0,01)$ entre las medias del ICT y los grupos de trabajadores por edad en todos los factores. Los trabajadores hasta 35 años presentaron una media del ICT de $40,83(\mathrm{dp} \pm 4,77)$; de 36 a 50 años, una media de $39,77(\mathrm{dp} \pm 5,26) ;$ y más de 50 años, una media de $39,04(\mathrm{dp} \pm 5,03)$. Los valores relativos al factor 1 y 3 aumentaron con la edad, mientras el factor 2 y la capacidad global disminuyeron con la edad (Tabla 3). En la validez convergente o discriminante, los factores extraídos del ICT presentaron correlaciones estadísticamente significativas $(p<0,05)$

Tabla 2. Análisis de los componentes principales y consistencia interna del índice de capacidad para el trabajo. Bahía, 2011-2012.

\begin{tabular}{|c|c|c|c|c|c|c|}
\hline Pregunta & Factor 1 & Factor 2 & Factor 3 & Comunalidad & Error & $\begin{array}{c}\text { Alfa de } \\
\text { Cronbach }\end{array}$ \\
\hline 1. Capacidad para el trabajo actual & - & 0,46 & - & 0,38 & 0,62 & 0,61 \\
\hline 2. Exigencias físicas del trabajo & - & 0,77 & - & 0,63 & 0,37 & 0,41 \\
\hline 3. Exigencias mentales del trabajo & - & 0,90 & - & 0,71 & 0,29 & 0,43 \\
\hline 4. Número de enfermedades & - & - & 0,75 & 0,57 & 0,43 & 0,35 \\
\hline 5. Faltas al trabajo debido a enfermedades & - & - & 0,74 & 0,57 & 0,43 & 0,35 \\
\hline 6. Ausentismo & - & - & 0,64 & 0,37 & 0,63 & 0,47 \\
\hline 7. Pronóstico de la capacidad para el trabajo & - & - & 0,31 & 0,24 & 0,76 & 0,48 \\
\hline 8. Valoración de las actividades diarias & 0,82 & - & - & 0,65 & 0,35 & 0,61 \\
\hline 9. Sentirse activo y alerta & 0,79 & - & - & 0,68 & 0,32 & 0,57 \\
\hline 10. Esperanza para el futuro & 0,79 & - & - & 0,58 & 0,42 & 0,71 \\
\hline Varianza del componente (\%) & 29,0 & 13,8 & 11,0 & - & - & - \\
\hline Varianza total acumulada (\%) & 53,9 & - & - & - & - & - \\
\hline \multicolumn{7}{|l|}{ Correlación } \\
\hline Factor $1-$ Factor 2 & 0,365 & - & - & - & - & - \\
\hline Factor 1 - Factor 3 & 0,241 & - & - & - & - & - \\
\hline Factor 2 - Factor 3 & 0,303 & - & - & - & - & - \\
\hline \multicolumn{7}{|l|}{ Consistencia interna } \\
\hline Omega de McDonald & 0,84 & 0,78 & 0,72 & - & - & - \\
\hline Alfa de Cronbach & 0,72 & 0,61 & 0,53 & - & - & - \\
\hline
\end{tabular}


Tabla 3. Comparación entre el índice de capacidad para el trabajo según grupos de trabajadores por edad. Bahia, Brasil, 2011-2012.

\begin{tabular}{|c|c|c|c|c|c|c|c|c|}
\hline \multirow{2}{*}{ Factor/ICT } & \multirow{2}{*}{ Puntuación } & \multicolumn{2}{|c|}{$<35$ años } & \multicolumn{2}{|c|}{ 36-50 años } & \multicolumn{2}{|c|}{$>50$ años } & \multirow{2}{*}{$\begin{array}{l}\text { Valor } \\
\text { de } p^{*}\end{array}$} \\
\hline & & Media & DE & Media & DE & Media & DE & \\
\hline 1. Recursos mentales & $1-4$ & 3,40 & 0,74 & 3,59 & 0,67 & 3,66 & 0,59 & 0,01 \\
\hline 2. Capacidad para el trabajo física y mental & $2-20$ & 16,41 & 2,10 & 16,10 & 2,33 & 16,28 & 2,07 & 0,01 \\
\hline $\begin{array}{l}\text { 3. Presencia de enfermedades y } \\
\text { restricciones por el estado de salud }\end{array}$ & $7-25$ & 21,00 & 3,33 & 20,01 & 3,69 & 19,07 & 3,77 & 0,01 \\
\hline Índice de capacidad para el trabajo & $7-49$ & 40,83 & 4,77 & 39,77 & 5,25 & 39,04 & 5,03 & 0,01 \\
\hline
\end{tabular}

con las escalas de salud física y mental y los estresores ocupacionales, excepto el factor 2 con demanda psicológica en el trabajo $(p>0,05)$. Hubo correlación positiva similar con el control sobre el trabajo entre los tres factores, el apoyo social fue mayor con el factor $1(r=0,12)$ y la autopercepción del estado de salud con el factor $3(r=0,40)$. La correlación negativa fue ascendente entre la demanda psicológica y el factor $1(r=-0,13)$

Tabla 4. Correlación entre los factores extraídos del índice de capacidad para el trabajo según constructos de salud física, mental y estresores ocupacionales. Bahía, Brasil, 2011-2012.

\begin{tabular}{llll} 
Escalas de salud física & \multicolumn{3}{c}{ Correlación de Spearman* } \\
\cline { 2 - 4 } $\begin{array}{l}\text { y mental y aspectos } \\
\text { psicosociales }\end{array}$ & Factor 1 & Factor 2 & Factor 3
\end{tabular}

\begin{tabular}{|c|c|c|c|}
\hline $\begin{array}{l}\text { Autopercepción del estado } \\
\text { de salud }\end{array}$ & 0,23 & 0,36 & 0,40 \\
\hline $\begin{array}{l}\text { Apoyo social de los } \\
\text { colegas y directivos }\end{array}$ & 0,12 & 0,10 & 0,07 \\
\hline Control en el trabajo & 0,12 & 0,13 & 0,12 \\
\hline $\begin{array}{l}\text { Demanda psicológica en } \\
\text { el trabajo }\end{array}$ & $-0,13$ & $-0,03$ & $-0,07$ \\
\hline Demanda física del trabajo & $-0,11$ & $-0,14$ & $-0,20$ \\
\hline Demanda emocional & $-0,08$ & $-0,07$ & $-0,12$ \\
\hline $\begin{array}{l}\text { Desequilibrio esfuerzo- } \\
\text { recompensa }\end{array}$ & $-0,10$ & $-0,14$ & $-0,16$ \\
\hline $\begin{array}{l}\text { Trastornos mentales } \\
\text { comunes }\end{array}$ & $-0,35$ & $-0,33$ & $-0,44$ \\
\hline
\end{tabular}

y entre el factor 3 con las demandas física $(r=-0,20)$ y emocional $(r=-0,12)$, desequilibrio esfuerzo-recompensa $(r=-0,16)$ y los trastornos mentales comunes $(r=-0,44)$. Todas las correlaciones fueran débiles, excepto la autopercepción del estado de salud y trastornos mentales comunes que presentaron correlación positiva y negativa moderada, respectivamente (Tabla 4).

La consistencia interna del ICT fue mensurada por el coeficiente alfa de Cronbach y por el omega de McDonald, y se obtuvieron en cada factor, respectivamente: $1^{\circ}$ factor 0,72 y 0,$84 ; 2^{\circ}$ factor 0,61 y 0,78 ; y el $3^{\circ}$ factor 0,53 y 0,72 (Tabla 2 ).

\section{DISCUSIÓN}

En el presente estudio se evaluaron los componentes (factores), la consistencia interna y la discriminación del ICT entre trabajadores de la salud de la atención de baja y media complejidad. Las preguntas del ICT presentaron sensibilidad psicométrica, se extrajeron tres factores del instrumento con varianza total acumulada superior al 50\%. La mayoría de los ítems presentó un poder explicativo compatible con el factor y los datos referentes a la consistencia interna de los factores se presentaron regulares. EI ICT mostró diferencias en las puntuaciones medias de los factores evaluados en función de los grupos de 
edad y hubo correlaciones entre los factores extraídos del ICT y aspectos de la salud física, mental y psicosocial del trabajo.

El análisis de los componentes principales indicó la reducción de las preguntas del ICT en tres factores que explican los recursos mentales, la capacidad para el trabajo física y mental y la presencia de enfermedades y restricción ante el estado de salud. Este modelo confirma el concepto multidimensional de la capacidad para el trabajo con relación a los aspectos físicos y mentales del trabajador ${ }^{(1)}$. A pesar de que el ICT esté compuesto por diez preguntas que se organizan en siete dimensiones, la no extracción de los siete factores previstos en su base teórica puede estar relacionado, dado que presentaron conceptos que interactúan entre sí.

La extracción de tres factores del ICT, como fue observado en el presente estudio, fue confirmado por otros estudios realizados en Brasil y en otros países. Un estudio factorial confirmatorio realizado con trabajadores del sector eléctrico en Brasil(8) presentó una dimensionalidad similar, pero la varianza total acumulada fue mayor, con el 57,9\%. Otros estudios ${ }^{(11,12)}$ presentaron tres factores dimensionales, ambos presentaron semejanzas a lo encontrado en este estudio solo en el tercer factor. Otro estudio realizado con enfermeras de países de Europa ${ }^{(10)}$ presentó dos factores en siete países y un factor en dos países. Se observa que los estudios se diferencian con relación a la cantidad de factores extraídos y las preguntas que se agregan en cada factor. Estas diferencias están relacionadas tanto con las características metodológicas del estudio, como con la variabilidad de aplicación del ICT en diferentes países.

La varianza total acumulada confiere mayor poder explicativo a los factores en el siguiente orden: recursos mentales, capacidad para el trabajo física y mental y presencia de enfermedades y restricción ante el estado de salud. Se observa que el factor recursos mentales contribuye con una mayor proporción explicativa de la capacidad para el trabajo entre trabajadores de la salud, pues se refiere a la reducción de los aspectos cognitivos, perceptivos, de memoria, aprendizaje, pen- samiento y velocidad del procesamiento de la información ${ }^{(24)}$. La salud mental fue uno de los aspectos de menor asociación con la capacidad para el trabajo, y fue más relevante en el contexto laboral con demandas mentales elevadas ${ }^{(3)}$, situación que puede estar relacionada con el contexto de los trabajadores de la salud.

EI ICT presentó diferencias entre grupos de trabajadores por edad: menores de 35 años, 36 a 50 años y mayores de 50 años, con un descenso de las puntuaciones entre los de mayor edad. En el primer factor (recursos mentales) se observó un aumento de las puntuaciones con el envejecimiento cronológico, lo que puede ser explicado por el hecho de que la edad, la experiencia ocupacional, la adaptación al ambiente de trabajo y también la mejor forma de hacer frente a los conflictos laborales están influenciados por el desarrollo mental.

En el segundo factor (capacidad para el trabajo física y mental) se observó una reducción de las puntuaciones en el grupo de trabajadores entre 36 y 50 años, al ser comparado con el grupo de más de 50 años. En el tercer factor (presencia de enfermedades y restricción ante el estado de salud) se observó una disminución de las puntuaciones con el envejecimiento cronológico, lo cual era esperado por el número de enfermedades entre los de mayor edad.

De este modo, se percibe que el envejecimiento cronológico puede estar vinculado a la sobrecarga de trabajo y a una mayor exposición a las condiciones precarias de trabajo a lo largo del tiempo, lo que favorece la reducción de la capacidad para el trabajo. Ciertos estudios con trabajadores han señalado que, cuanto mayor es la edad, las puntuaciones del ICT son menores, o sea, el envejecimiento cronológico influye en la reducción de la capacidad para el trabajo $^{(25,26,27)}$, si las actividades desarrolladas en el trabajo dependen de la capacidad fisiológica $^{(28)}$. Si bien la edad interfiere en la salud, los jóvenes también se enferman y no todas las enfermedades comprometen la capacidad funcional, dado que las personas pueden trabajar, incluso con enfermedades, más allá de 
que algunas enfermedades sean evitables ${ }^{(24)}$. Así, la capacidad para el trabajo puede tornarse insatisfactoria a lo largo de la vida no solo por el envejecimiento fisiológico, sino por el envejecimiento funcional relacionado con aspectos sociodemográficos, estilo de vida y exigencias del trabajo ${ }^{(29)}$.

Frente a estos resultados, se proponen recomendaciones internacionales para el desarrollo de estudios sobre la capacidad para el trabajo con trabajadores a partir de 35 años en los países en desarrollo, pues en estos países las condiciones de vida y de trabajo son menos favorables ${ }^{(30)}$ y por eso es indispensable iniciar esta evaluación con trabajadores más jóvenes a fin de establecer estrategias de prevención, manutención y promoción de la capacidad para el trabajo.

Los factores extraídos del ICT estuvieron correlacionados a la salud física de forma positiva con la autopercepción del estado de salud, y negativa con la demanda física en el trabajo, lo que fue ratificado por un estudio realizado con trabajadores de la salud de atención primaria, en el que el ICT presentó mayores puntuaciones entre las dimensiones de la salud física de la calidad de vida ${ }^{12}$. En cuanto a la salud mental y a los aspectos psicosociales del trabajo, los factores del ICT presentaron correlación positiva con el control en el trabajo y el apoyo social, y correlación negativa con la demanda psicológica y emocional, con los trastornos mentales comunes y con el estrés en el trabajo (desequilibrio esfuerzo-recompensa). Estudios con trabajadores de enfermería ${ }^{(5,10)}$ confirman estos hallazgos, en los que el ICT presentó correlación positiva con el control en el trabajo y la recompensa, y correlación negativa con el burnout, con la demanda psicológica y con el esfuerzo.

Al considerar la base teórica de la capacidad para el trabajo, las correlaciones presentadas fueron las esperadas, ya que los trabajadores que estaban con su estado de salud físico y mental comprometido también presentaron reducción de la capacidad para el trabajo; los trabajadores que tenían mayor soporte social y mayor control sobre su trabajo presentaron mayor capacidad para el trabajo en los tres factores; y los trabajadores que consideraron su estado de salud bueno y muy bueno tenían una mayor capacidad para el trabajo en los tres factores. Estas correlaciones confirman el concepto del ICT como un índice que propone identificar la autopercepción del trabajador con relación a la capacidad para el trabajo en función de las exigencias, de su estado de salud y de sus capacidades físicas y mentales ${ }^{(1)}$.

Los resultados del alfa de Cronbach señaIaron una consistencia interna baja en la tercera dimensión (presencia de enfermedades y restricción ante el estado de salud), aceptable en la segunda dimensión (capacidad para el trabajo física y mental), y buena en la primera dimensión (recursos mentales). Ciertos estudios de validez del ICT han presentado un alfa de Cronbach total con relación a todos los ítems del cuestionario y los valores se condicen con una consistencia interna del instrumento entre buena y óptima ${ }^{(4)}$, variando de $0,72^{(8,10)}$ a $0,80^{(12,22)}$.

Sin embargo, el uso de todos los ítems para evaluar la consistencia interna es recomendado solo para cuestionarios que presentan solo un factor. Pues, el número de preguntas de un factor influye en el valor del alfa, por lo que se esperan valores menores en factores con un pequeño número de preguntas. De cualquier forma, los factores se componen para indicar una determinada dimensión de intereses y la opción de factores con pequeño número de preguntas puede no ser apropiada por la baja consistencia interna. Este aspecto ayuda a reflexionar sobre los factores que, de hecho, estructuran el ICT como un índice.

Al considerar las limitaciones del alfa de Cronbach ${ }^{(21)}$, se estimó también el omega de McDonald, y se comprobó que el alfa presentó medidas subestimadas. Así, la comparación entre los dos coeficientes lleva a medidas más precisas, para lo cual es necesario comparar los dos coeficientes y la mayor medida que se podría obtener. Valores aceptables de la consistencia interna son considerados aquellos $>0,60$, lo que fue observado en el presente estudio en los factores 1 y 2 con el alfa de Cronbach y en los tres factores con el 
omega. Solo un estudio con trabajadores de enfermería ${ }^{(5)}$ estimó el omega entre los ítems del ICT $(0,87)$ y si bien se observó una consistencia interna óptima, incluyeron en la evaluación de esa consistencia tan solo un factor.

Desde el punto de vista teórico, una de las limitaciones del instrumento se refiere a la identificación de factores relacionados con los aspectos del trabajo que interfieren en la capacidad para el trabajo. EI ICT fue elaborado con el soporte teórico del modelo estrés-desgaste, el cual considera que los recursos individuales del trabajador median la relación entre el estrés y el desgaste en el trabajo $^{(2)}$. En este modelo, las herramientas y el ambiente de trabajo forman parte de las fuentes de estresores, por lo que estos constructos podrían haber sido incluidos en el ICT ${ }^{(12)}$. Además, el uso del análisis por factores del ICT es una limitación del instrumento, al considerar que su propuesta teórica sustenta su uso como un índice sin subdivisiones.

Las limitaciones de este estudio se refieren tanto al efecto del trabajador sano -al considerar que aquellos trabajadores apartados del cargo podrían presentar una capacidad para el trabajo comprometida- como a los puntos de corte utilizados para caracterizar la población por el ICT, cuyos criterios no están validados en Brasil, limitación ya citada por otros autores ${ }^{(6,8,27)}$. Si bien el no uso de la matriz de correlación policórica puede haber sido una limitación del análisis, las escalas ordinales, politómicas y discretas del instrumento no hieren la sensibilidad psicométrica devido al tamaño muestral, el cual fue considerado excelente para el análisis y la distribución de las variables. A pesar de las limitaciones, este estudio realizó análisis que aún no habían sido presentados en los estudios anteriores, como la evaluación del ICT entre grupos de trabajadores por franja etária (edad) y de acuerdo con los indicadores de salud física y mental y estresores ocupacionales y, también, estimar diferentes coeficientes para evaluar la consistencia interna por factores (incluyendo el omega de McDonald). Por lo tanto, presenta análisis de indicadores nuevos que permitieron evaluación más amplia del desempeño del ICT, que contribuye a una mayor visibilidad de las potencialidades y limitaciones de este instrumento.

\section{CONCLUSIÓN}

Aunque el ICT no se proponga evaluar la capacidad para el trabajo por cada factor, la concepción que sustenta su propuesta se asienta en una perspectiva multidimensional, tal como fue propuesto por los investigadores que lo elaboraron, quienes consideraron siete dimensiones teóricas. En este estudio, esta estructura no fue confirmada, y se obtuvieron solo tres factores. No obstante, a pesar de obtener un número menor de factores que el propuesto, a través de los parámetros estadísticos presentados, se puede decir que el instrumento presentó propiedades psicométricas satisfactorias tanto para medir la capacidad para el trabajo físico y mental como para identificar grupos de trabajadores por edad y correlacionarlos con los aspectos de la salud física, mental y estresores ocupacionales.

De ese modo, los hallazgos orientarían el uso del ICT en futuras investigaciones y en las organizaciones de trabajo con poblaciones similares a las del presente estudio con el fin de prevenir la incapacidad, mantener $y / o$ promover la capacidad para el trabajo entre trabajadores de la salud de Brasil. Se necesita invertir en futuros estudios que aborden el análisis de test y retest, el análisis factorial confirmatorio, el análisis factorial múltiple, el análisis de criterios de clasificación y el análisis de cargas cruzadas que podrían contribuir con otras medidas psicométricas de evaluación del ICT. 


\section{AGRADECIMENTOS}

Al Conselho Nacional de Desenvolvimento Científico e Tecnológico (480611/2010-6) y a la Fundação de Amparo à Pesquisa do Estado da Bahia (SUS0024/2009) y la beca de maestría (BOL0423/2013) por el apoyo financiero.

\section{REFERENCIAS BIBLIOGRÁFICAS}

1. Tuomi K, Ilmarinen J, Janhkola A, Katajarinne L, Tulkki A. Índice de capacidade para o trabalho. Fischer FM, traductora. São Carlos: EdUFSCar; 2005.

2. Ilmarinen J, Tuomi K, Eskelinen L, Nygard CH, Huuhtanen P, Klockars M. Background and objectives of the Finnish research project on aging works in municipal occupations. Scandinavian Journal of Work, Environment and Health. 1991;17(Suppl 1):7-11.

3. Martinez MC, Latorre MRDO, Fischer FM. Capacidade para o trabalho: revisão de literatura. Ciência \& Saúde Coletiva. 2010;15(Suppl 1):15531561.

4. Cordeiro TMSC, Souza DS, Araújo TM. Validade, reprodutibilidade e confiabilidade do índice de capacidade para o trabalho: uma revisão sistemática. Revista de Epidemiologia e Controle de Infecção. 2017;7(1):57-66.

5. Silva Júnior SHA, Vasconcelos AGG, Griep RH, Rotenberg L. Validade e confiabilidade do índice de capacidade para o trabalho (ICT) em trabalhadores de enfermagem. Cadernos de Saúde Pública. 2011;27(6):1077-1087.

6. Silva Júnior SHA, Vasconcelos AGG, Griep RH, Rotenberg L. Confiabilidade teste-reteste do índice de capacidade para o trabalho (ICT) em trabalhadores de enfermagem. Revista Brasileira de Epidemiologia. 2013;16(1):202-209.

7. Renosto A, Biz P, Hennington EA, Pattussi MP. Confiabilidade teste-reteste do índice de capacidade para o trabalho em trabalhadores metalúrgicos do Sul do Brasil. Revista Brasileira de Epidemiologia. 2009;12(2):217-225.

8. Martinez MC, Latorre MRDO, Fischer FM. Validity and reliability of the brazilian version of the work ability index questionnaire. Revista de Saúde Pública. 2009;43(3):525-532.

9. Zwart $\mathrm{BCH}$, Frings-Dresen MHW, Duivenbooden JC. Test-retest reliability of the work ability index questionnaire. Occupational Medicine. 2002;52(4):177-181.

10. Radkiewicz P, Bazyl-Widerszal M. Psychometric properties of work ability index in the light of comparative survey study. International Congress Series. 2005;1280:304-309.

11. Abdolalizadeh $M$, Arastoo AA, Ghsemeadel R, Montazeri A, Ahmadi K, Azizi A. The psychometric properties of an iranian translation of the work ability index (WAI) questionnaire. Journal of Occupational Rehabilitation. 2012;22(3):401-408.

12. Peralta N, Vasconcelos AGG, Griep RH, Miller L. Validez y confiabilidad del índice de capacidad para el trabajo en trabajadores del primer nivel de atención de salud en Argentina. Salud Colectiva. 2012;8(2):163-173.

13. Alexopoulos EC, Merekoulias G, Gnardellis C, Jelastopulu E. Work ability index: validation of the greek version and descriptive data in heavy industry employees. British Journal of Medicine and Medical Research. 2013;3(3):608-621.

14. Yang DJ, Kang D, Kim YK, Kim YH, Yang YA, Cha SM, Eom IK, Kim JE. Reliability of self-administered work ability index questionnaire among korean workers. Ergonomics. 2013;56(11):16521657.

15. Martus $P$, Jakob O, Rose $U$, Seibt R, Freude G. A comparative analysis of the work ability index. Occupational Medicine. 2010;60(7):517-524.

16. Santos KOB, Araújo TM, Pinho PS, Silva ACC. Avaliação de um instrumento de mensuração de morbidade psíquica: estudo de validação do selfreporting questionnaire (SRQ-20). Revista Baiana de Saúde Pública. 2010;34(3):544-560.

17. Siegrist J. Adverse health effects of high-effortlow reward conditions at work. Journal of Occupational Health Psychology. 1996;1(1):27-41.

18. Araújo TM, Karasek R. Validity and reliability of the job content questionnaire in formal and informal Jobs in Brazil. SJWEH Supplements. 2008;(6):52-59.

19. Karasek RA. Job content questionnaire and user's guide. Lowell: University of Massachusetts; 1985.

20. Damásio BF. Uso da análise fatorial exploratória em psicologia. Avaliação Psicológica. 2012;11(2): 213-228.

21. Collares CF, Grec WLP, Machado JLM. Psicometria na garantia de qualidade da educação 
médica: conceitos e aplicações. Science in Health. 2012;3(1):33-49.

22. Hair Jr JF, Black WC, Babin BJ, Anderson RE. Multivariate Data Analysis. New Jersey: Prentice Hall; 1998.

23. Revelle W, Zinbarg RE. Coefficients alpha, beta, omega and the glb: comments os Sijtsma. Psychometrika. 2009;74(1):145-154.

24. Ilmarinen J. Towards a longer worklife!: ageing and the quality of worklife in the European Union. Helsinki: Finnish Institute of Occupational Health; 2005.

25. Hilleshein EF, Souza LM, Lautert L, Paz AA, Catalan VM, Teixeira MG, Mello DB. Capacidade para o trabalho de enfermeiros de um hospital universitário. Revista Gaúcha de Enfermagem. 2011;32(3):509-515.

26. Monteiro MS, Alexandre NMC, Milani D, Fujimara F. Work capacity evaluation among nursing aides. Revista da Escola de Enfermagem da USP. 2011;45(5):1177-1182.

27. Cordeiro TMSC, Araújo TM. Capacidade para o trabalho entre trabalhadores do Brasil. Revista Brasileira de Medicina do Trabalho. 2016;14(3): 262-274.

28. Sampaio RF, Augusto VG. Envelhecimento e trabalho: um desafio para a agenda da reabilitação. Brazilian Journal of Physical Therapy. 2012;16(2):94-101.

29. Silva LG, Haddad MCL, Domansky RC, Vituri DW. Capacidade para o trabalho entre trabalhadores de higiene e limpeza de um hospital universitário público. Revista Eletrônica de Enfermagem. 2010;12(1):158-163.

30. Bellusci SM, Fischer FM. Envelhecimento funcional e condições de trabalho em servidores forenses. Revista de Saúde Pública. 1999;33(6): 602-609.

FORMA DE CITAR

Cordeiro TMSC, Araújo TM, Santos KOB. Estudio exploratorio de validez y consistencia interna del índice de capacidad para el trabajo entre trabajadores de la salud. Salud Colectiva. 2018;14(4):713-724. doi: 10.18294/sc.2018.1342.

Recibido: 3 de abril de 2017 | Versión final: 13 de noviembre de 2017 | Aprobado: 15 de diciembre de 2017

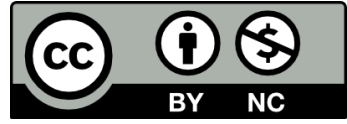

Este obra está bajo una licencia de Creative Commons Reconocimiento-NoComercial 4.0 Internacional. Reconocimiento - Permite copiar, distribuir y comunicar públicamente la obra. A cambio, se debe reconocer y citar al autor original. No Comercial - Esta obra no puede ser utilizada con finalidades comerciales, a menos que se obtenga el permiso.

http://dx.doi.org/10.18294/sc.2018.1342

Este artículo fue traducido del portugués por Viviana Martinovich. 